The occurrence of the exponent $2 / m$, where $1 / m$ is found in the case of functions of a single variable, is due to the fact that the magnitude of an interval of length $1 / n$ is replaced in the course of the present reasoning by the area of a square having a quantity of the order of $1 / n$ for the length of its side.

This result is significant only if $m>2$, since otherwise the condition as stated requires that $f(x, y)$ be constant. When $m=2$, it is sufficient (in consequence of III) that $f(x, y)$ have continuous first partial derivatives. There are corresponding results for $m<2$.

The reasoning is not materially changed if the $m$ th power in the integral to be minimized is multiplied by a positive measurable weight function $\rho(x, y)$ with a positive lower bound.

The University of Minnesota

\title{
ON A TENSOR OF THE SECOND RANK IN FUNCTION SPACE*
}

\section{BY DUNHAM JACKSON}

In a recent paper $\dagger$ the writer has discussed a doubly infinite matrix of derivatives which has the properties of a tensor of the second rank in function space, and a quantity obtained by contraction of this tensor, which is analogous to a divergence. The latter concept is suggested formally by the writing of an infinite series, the general term of which in many cases does not approach zero; but an example was constructed in which the series is convergent, and defines a quantity which can be alternatively expressed in a form independent of any particular coordinate system.

* Presented to the Society, September 9, 1926.

$\dagger \mathrm{D}$. Jackson, Some convergence proofs in the vector analysis of function space, Annals of Mathematics, vol. 27, pp. 551-567. 
The purpose of this note is to point out that the tensor of the second rank may appropriately be identified with a certain double series, or with the function of two variables represented by the series, and that this immediately leads to a simplified invariant interpretation of the divergence.

The notation and hypotheses of the earlier paper will be retained. The relation

$$
\Phi(x)=\int_{0}^{2 \pi} K(x, t) \phi[f(t)] d t
$$

defines what may be regarded as an example of a vector point function in function space, with $f(x)$ as independent variable. The functions

$$
u_{n}(x), \quad(n=1,2, \cdots),
$$

form a normalized orthogonal system, constructed in a particular way, and having properties which were discussed in some detail in the paper cited. If $a_{n}$ and $A_{n}$ are the coefficients of $u_{n}(x)$ in the developments of $f(x)$ and $\Phi(x)$ respectively according to the system of $u$ 's, each $A_{i}$ is a function of $a_{1}, a_{2}, \cdots$, and the general component of the tensor in question is

$$
\frac{\partial A_{i}}{\partial a_{j}}=\int_{0}^{2 \pi} \int_{0}^{2 \pi} K(x, t) \phi^{\prime}[f(t)] u_{i}(x) u_{j}(t) d t d x .
$$

The point to be made in the present note is that the last expression is the coefficient of $u_{i}(x) u_{j}(t)$ in the formal expansion of*

$$
K(x, t) \phi^{\prime}[f(t)]
$$

in a double series of the $u$ 's. Since it is known furthermore under the hypotheses of $\S 8$ of the paper cited that

$$
\left|\frac{\partial A_{i}}{\partial a_{j}}\right| \leqq \frac{Q}{i^{2} j^{2}},
$$

\footnotetext{
* I am indebted to Dr. A. D. Michal for the observation, which had escaped me when the note was presented to the Society, that for each value of $x$ the expression (1) is the functional derivative of the functional $\Phi$.
} 
where $Q$ is independent of $i$ and $j$, and since $\left|u_{n}(x)\right|$ does not exceed a constant multiple of $n^{1 / 2}$, the typical form of the series satisfies an inequality of the form

$$
\left|\frac{\partial A_{i}}{\partial a_{j}} u_{i}(x) u_{j}(t)\right| \leqq \frac{Q^{\prime}}{i^{3 / 2} j^{3 / 2}},
$$

and the series converges uniformly, inasmuch as the double series having the quantity on the right for its general term is convergent. A familiar line of argument shows that the sum of the series is necessarily the function (1). This function of two variables may therefore be taken as an invariant representation of the tensor, while the expansion in series indicates its resolution into components according to the orthogonal system.

Termwise integration of the series for $K(t, t) \phi^{\prime}[f(t)]$, with due regard to the orthogonality of the $u$ 's, leads to the relation

$$
\sum_{i} \frac{\partial A_{i}}{\partial a_{i}}=\int_{0}^{2 \pi} K(t, t) \phi^{\prime}[f(t)] d t
$$

which expresses the "divergence" in a form independent of the set of $u$ 's taken as a system of reference. The form given in the earlier paper, in terms of double integrals, can be reduced to this one by integration by parts.

The University of Minnesota 